\title{
ARBITRARY TRIPLE SYSTEMS ADMITTING A MULTIPLICATIVE BASIS
}

\author{
ANTONIO J. CALDERÓN, FRANCISCO J. NAVARRO, AND JOSÉ M. SÁNCHEZ
}

\begin{abstract}
Let $(T,\langle\cdot, \cdot, \cdot\rangle)$ be a triple system of arbitrary dimension, over an arbitrary base field $\mathbb{F}$ and in which any identity on the triple product is not supposed. A basis $\mathcal{B}=$ $\left\{e_{i}\right\}_{i \in I}$ of $T$ is called multiplicative if for any $i, j, k \in I$ we have that $\left\langle e_{i}, e_{j}, e_{k}\right\rangle \in \mathbb{F} e_{r}$ for some $r \in I$. We show that if $T$ admits a multiplicative basis then it decomposes as the orthogonal direct sum $T=\bigoplus \mathfrak{I}_{k}$ of well-described ideals admitting each one a multiplicative basis. Also the minimality of $T$ is characterized in terms of the multiplicative basis and it is shown that, under a mild condition, the above direct sum is by means of the family of its minimal ideals.

Keywords: Multiplicative basis, triple system, non-associative triple system, infinite dimensional triple system, structure theory.

2010 MSC: 15A03, 17A40, 17A60.
\end{abstract}

\section{INTRODUCTION AND PREVIOUS DEFINITIONS}

Throughout this paper $T$ will denote an arbitrary triple system in the sense that there are not restrictions on the dimension of $T$ or on the base field $\mathbb{F}$, and that any identity on the triple product (associativity, alternativity, Lie, Jordan, etc.) is not supposed. That is, $T$ is just a linear space over $\mathbb{F}$ endowed with a trilinear map

called the triple product of $T$.

$$
\begin{gathered}
T \times T \times T \rightarrow T \\
(x, y, z) \mapsto\langle x, y, z\rangle
\end{gathered}
$$

Definition 1.1. A basis $\mathcal{B}=\left\{e_{i}\right\}_{i \in I}$ of $T$ is said to be multiplicative if for any $i, j, k \in I$ we have either $\left\langle e_{i}, e_{j}, e_{k}\right\rangle=0$ or $0 \neq\left\langle e_{i}, e_{j}, e_{k}\right\rangle \in \mathbb{F} e_{r}$ for some (unique) $r \in I$.

Remark 1.1. The definition of multiplicative basis given in Definition 1.1 is a little bit more general than the usual one considered in the literature for the case of algebras ([4, $6,7,8,21])$. In fact, in these references, a basis $\mathcal{B}=\left\{e_{i}\right\}_{i \in I}$ of an algebra $A$ is called multiplicative if for any $i, j \in I$ we have either $e_{i} e_{j}=0$ or $0 \neq e_{i} e_{j}=e_{k}$ for some $k \in I$.

To construct examples of triple systems admitting multiplicative bases we just have to fix an arbitrary (non-empty) set of indexes $I$ and two arbitrary mappings $\alpha: I \times I \times I \rightarrow I$ and $\beta: I \times I \times I \rightarrow \mathbb{F}$. Then the $\mathbb{F}$-linear space $T$ with basis $\mathcal{B}=\left\{e_{i}\right\}_{i \in I}$ and product among the element of the basis given by $\left\langle e_{i}, e_{j}, e_{k}\right\rangle=\beta(i, j, k) e_{\alpha(i, j, k)}$ becomes a triple system admitting $\mathcal{B}$ as a multiplicative basis.

Given an algebra $A$, with product denoted by juxtaposition, we can endow the underlying linear space of $A$ with a structure of triple system $T_{A}$ by defining the triple product as $\langle x, y, z\rangle=(x y) z$. We say that $T_{A}$ is the triple system associated to $A$. We have that if $A$

Supported by the PCI of the UCA 'Teoría de Lie y Teoría de Espacios de Banach', by the PAI with project numbers FQM298, FQM7156 and by the project of the Spanish Ministerio de Educación y Ciencia MTM201015223. 
admits a multiplicative algebra basis (in our extended sense), that is, a basis $\mathcal{B}=\left\{e_{i}\right\}_{i \in I}$ such that for any $i, j \in I$ we have either $e_{i} e_{j}=0$ or $0 \neq e_{i} e_{j} \in \mathbb{F} e_{r}$, for some $r \in I$, then $T_{A}$ becomes a triple systems admitting $\mathcal{B}$ as a multiplicative basis. From here, since it is usual in the literature to describe an algebra by exhibiting a multiplicative table among the elements of a fixed basis, we can find many classical examples of triple systems admitting multiplicative bases in the categories of associative triple systems, alternative triple systems, Lie triple systems, Jordan triple systems, Leibniz triple systems and so on. For instance, in the category of associative triple systems we have that the classes of triple systems associated to full matrix algebras, to group-algebras, to quiver algebras (when $\mathbb{F}$ is algebraically closed) or to finite-dimensional associative algebras of finite representation type, ([3, 4, 5, 6, 19, 21, 23]), are examples of (associative) triple systems admitting multiplicative basis. In the category of Lie triple systems we can take as examples of triple systems admitting multiplicative basis the ones associated to semisimple finite-dimensional Lie algebras (over algebraically closed fields of characteristic 0), to semisimple separable $L^{*}$-algebras [24], to semisimple locally finite split Lie algebras [25], to Heisenberg algebras [22], to twisted Heisenberg algebras [1] or to the split Lie algebras studied in [9, $\S 3]$.

Consider now the associative triple system $\mathcal{M}_{n \times m}(\mathbb{F})$ where the triple product is given by $\langle A, B, C\rangle:=\left(A B^{t}\right) C$, (denoting by $B^{t}$ the transpose of $\left.B\right)$. Then $\left(\mathcal{M}_{n \times m}(\mathbb{F}),\langle\cdot, \cdot, \cdot\rangle\right)$ admits the standard basis $\mathcal{B}=\left\{E_{i, j}: 1 \leq i \leq n, 1 \leq j \leq m\right\}$ of $\mathcal{M}_{n \times m}(\mathbb{F})$ as a multiplicative basis. Following the terminology of [20], the class of Jordan or Lie triple systems formed for those triple systems called meson triples, (by their applications in the theory of meson fields), are also examples of triple systems admitting a multiplicative basis. We recall that this family of triple systems are constructed as follows. Take the linear subspace $\mathcal{M} \subset \mathcal{M}_{(n+1) \times(n+1)}(\mathbb{F})$ linearly generated by the matrices $G_{i}:=E_{i, n+1}-$ $E_{n+1, i}, i=1,2, \ldots, n$, and define the nonzero triple products among the elements of the basis as $\left\{G_{i}, G_{j}, G_{k}\right\}:=-\delta_{i j} G_{k}-\delta_{k j} G_{i}$ in the Jordan case, or $\left[\left[G_{i}, G_{j}\right], G_{k}\right]:=$ $\delta_{k i} G_{j}-\delta_{k j} G_{i}$ in the Lie case. In the same reference [20], we can find the family of Jordan and Lie triple systems defined as the linear subspaces $\mathcal{N} \subset \mathcal{M}_{(n+1) \times(n+1)}(\mathbb{F})$ linearly generated by the matrices $X_{i}:=E_{i, n+1}, Y_{i}:=E_{n+1, i}$ and where the nonzero triple products among the elements of the basis are defined by

$$
\left\{X_{i}, Y_{j}, X_{k}\right\}:=\delta_{j k} X_{i}+\delta_{j i} X_{k}, \quad\left\{Y_{i}, X_{j}, Y_{k}\right\}:=\delta_{j k} Y_{i}+\delta_{j i} Y_{k}
$$

in the Jordan case, or

$$
\left[\left[X_{i}, Y_{j}\right], X_{k}\right]:=\delta_{j k} X_{i}+\delta_{j i} X_{k}, \quad\left[\left[Y_{i}, X_{j}\right], Y_{k}\right]:=\delta_{j k} Y_{i}+\delta_{j i} Y_{k}
$$

in the Lie case. These are examples of Lie and Jordan triple systems with a multiplicative basis.

We also have as examples of Lie triple systems with multiplicative basis the ones introduced in $[10,11,12,15]$. Finally, we observe that can also find examples of 3-Lie algebras and Leibniz triple systems admitting a multiplicative basis in [16] and [2, Example 2.1, Example 2.2, Example 2.3] respectively.

A subtriple $S$ of a triple system $T$ is a linear subspace such that $\langle S, S, S\rangle \subset S$. A linear subspace $\mathcal{I}$ of $T$ is called an ideal if $\langle\mathcal{I}, T, T\rangle+\langle T, \mathcal{I}, T\rangle+\langle T, T, \mathcal{I}\rangle \subset \mathcal{I}$. We also recall that two nonzero ideals $\mathcal{I}, \mathcal{J}$ of $T$ are called orthogonal if the condition

$$
\langle T, \mathcal{I}, \mathcal{J}\rangle+\langle\mathcal{I}, T, \mathcal{J}\rangle+\langle\mathcal{I}, \mathcal{J}, T\rangle+\langle T, \mathcal{J}, \mathcal{I}\rangle+\langle\mathcal{J}, T, \mathcal{I}\rangle+\langle\mathcal{J}, \mathcal{I}, T\rangle=0
$$


holds. A direct sum $\bigoplus_{j \in J} \mathcal{I}_{j}$ of non-zero ideals of $T$ is called orthogonal direct sum if for any $j, k \in J$ with $j \neq k$ we have that $\mathcal{I}_{j}$ and $\mathcal{I}_{k}$ are orthogonal ideals.

The present paper is devoted to the study of arbitrary triple systems admitting a multiplicative basis, by focussing on its structure. The paper is organized as follows. In $\S 2$, we develop connections techniques in the set of indexes $I$ of the multiplicative basis, so as to get a powerful tool for the study of this class of triple systems. By making use of these techniques we show that any triple system $T$ admitting a multiplicative basis is the orthogonal direct sum $T=\bigoplus_{k} \mathcal{I}_{k}$ with any $\mathcal{I}_{k}$ a well described ideal of $T$ admitting also a multiplicative basis. In $\S 3$ the minimality of $T$ is characterized in terms of the multiplicative basis and it is shown that, in case the basis is $\mu$-multiplicative, the above decomposition of $T$ is actually by means of the family of its minimal ideals.

\section{Connections in the indeXes Set. Decompositions}

In what follows $\mathcal{B}=\left\{e_{i}\right\}_{i \in I}$ denotes a multiplicative basis of a triple system $T, \mathcal{P}(I)$ the power set of $I$ and $S_{3}$ the group of all permutations of three elements.

For each $i \in I$, a new variable $\bar{i} \notin I$ is introduced and we denote by $\bar{I}:=\{\bar{i}: i \in I\}$ the set consisting of all these new symbols. We will also write $\bar{U}:=\{\bar{i}: i \in U\}$ for any $U \in \mathcal{P}(I)$, and $\overline{(\bar{i})}:=i$ for any $i \in I$.

For any any $\sigma \in S_{3}$ we introduce the next mappings which recover, in a sense, certain multiplicative relations among the elements of $\mathcal{B}$ :

$$
a_{\sigma}: I \times I \times I \rightarrow \mathcal{P}(I) \text { and } b_{\sigma}: I \times \bar{I} \times \bar{I} \rightarrow \mathcal{P}(I)
$$

defined by

$$
\begin{aligned}
& a_{\sigma}\left(i_{1}, i_{2}, i_{3}\right)=\left\{\begin{array}{lll}
\emptyset & \text { if } & \left\langle e_{i_{\sigma(1)}}, e_{i_{\sigma(2)}}, e_{i_{\sigma(3)}}\right\rangle=0 \\
\{j\} & \text { if } & 0 \neq\left\langle e_{i_{\sigma(1)}}, e_{i_{\sigma(2)}}, e_{i_{\sigma(3)}}\right\rangle \in \mathbb{F} e_{j} .
\end{array}\right. \\
& b_{\sigma}\left(i, \bar{j}_{2}, \bar{j}_{3}\right)=\left\{j_{1} \in I: a_{\sigma}\left(j_{1}, j_{2}, j_{3}\right)=i\right\} .
\end{aligned}
$$

We also introduce the map

$$
\mu: I \times(I \dot{\cup} \bar{I}) \times(I \dot{\cup} \bar{I}) \longrightarrow \mathcal{P}(I)
$$

given by

- $\mu(I, I, \bar{I})=\mu(I, \bar{I}, I)=\emptyset$.

- $\mu\left(i_{1}, i_{2}, i_{3}\right)=\bigcup_{\sigma \in S_{3}} a_{\sigma}\left(i_{1}, i_{2}, i_{3}\right)$ in case $i_{1}, i_{2}, i_{3} \in I$.

- $\mu\left(i, \bar{j}_{2}, \bar{j}_{3}\right)=\bigcup_{\sigma \in S_{3}} b_{\sigma}\left(i, \bar{j}_{2}, \bar{j}_{3}\right)$ in case $i \in I$ and $\bar{j}_{2}, \bar{j}_{3} \in \bar{I}$.

Remark 2.1. Observe that $\mu\left(i_{1}, i_{2}, i_{3}\right)=\mu\left(i_{\sigma(1)}, i_{\sigma(2)}, i_{\sigma(3)}\right)$ for any $\sigma \in S_{3}$ and any $i_{1}, i_{2}, i_{3} \in I$; and that $\mu\left(i, \bar{j}_{2}, \bar{j}_{3}\right)=\mu\left(i, \bar{j}_{3}, \bar{j}_{2}\right)$ for any $i \in I$ and $\bar{j}_{2}, \bar{j}_{3} \in \bar{I}$.

Lemma 2.1. Let $i, j \in I$ and $x, y \in I \dot{U} \bar{I}$ be. Then $i \in \mu(j, x, y)$ if and only if $j \in \mu(i, \bar{x}, \bar{y})$.

Proof. Taking into account $\mu(I, I, \bar{I})=\mu(I, \bar{I}, I)=\emptyset$ we just have two cases to consider. Let $i \in \mu(j, x, y)$ be and suppose $x, y \in I$. Then there exists $\sigma \in S_{3}$ such that $i=$ $a_{\sigma}(j, x, y)$ and so $j \in b_{\sigma}(i, \bar{x}, \bar{y}) \subset \mu(i, \bar{x}, \bar{y})$ as wished. In case $x, y \in \bar{I}$, then there is $\sigma \in S_{3}$ such that $i \in b_{\sigma}(j, x, y)$ and consequently $j=a_{\sigma}(i, \bar{x}, \bar{y}) \subset \mu(i, \bar{x}, \bar{y})$. 
Finally, we also define the mapping

$$
\phi: \mathcal{P}(I) \times(I \dot{\cup} \bar{I}) \times(I \dot{\cup} \bar{I}) \longrightarrow \mathcal{P}(I)
$$

as

$$
\phi(J, j, k)=\bigcup_{i \in J} \mu(i, j, k)
$$

for any $J \in \mathcal{P}(I)$ and $j, k \in I \dot{\cup} \bar{I}$.

Lemma 2.2. Let $J \in \mathcal{P}(I)$ and $j, k \in I \dot{\cup} \bar{I}$ be. Then $i \in \phi(J, j, k)$ if and only if $\phi(\{i\}, \bar{j}, \bar{k}) \cap J \neq \emptyset$.

Proof. We have $i \in \phi(J, j, k)$ if and only if there exists $x \in J$ such that $i \in \mu(x, j, k)$. By Lemma 2.1, this is equivalent to $x \in \mu(i, \bar{j}, \bar{k}) \subset \phi(\{i\}, \bar{j}, \bar{k})$ and so also equivalent to $x \in \phi(\{i\}, \bar{j}, \bar{k}) \cap J \neq \emptyset$.

Definition 2.1. Let $i$ and $j$ be distinct elements in $I$. We say that $i$ is connected to $j$ if there exists a subset $\left\{i_{1}, i_{2}, i_{3}, \ldots, i_{2 n+1}\right\} \subset I \dot{\cup} \bar{I}$ for some $n \geq 1$, such that the following conditions hold:

1. $i_{1}=i$.

2. $\phi\left(\left\{i_{1}\right\}, i_{2}, i_{3}\right) \neq \emptyset$, $\phi\left(\phi\left(\left\{i_{1}\right\}, i_{2}, i_{3}\right), i_{4}, i_{5}\right) \neq \emptyset$,

: $\phi\left(\phi\left(\ldots \phi\left(\left\{i_{1}\right\}, i_{2}, i_{3}\right) \ldots\right), i_{2 n-2}, i_{2 n-1}\right) \neq \emptyset$.

3. $j \in \phi\left(\phi\left(\ldots \phi\left(\left\{i_{1}\right\}, i_{2}, i_{3}\right) \ldots\right), i_{2 n}, i_{2 n+1}\right)$.

The subset $\left\{i_{1}, i_{2}, i_{3}, \ldots, i_{2 n+1}\right\}$ is called a connection from $i$ to $j$ and we accept $i$ to be connected to itself.

Our next goal is to show that the connection relation is an equivalence relation. Previously we show the next result.

Lemma 2.3. Let $\left\{i_{1}, i_{2}, i_{3}, \ldots, i_{2 n+1}\right\}$ be a connection from $i$ to $j$, where $n \geq 1$ and $i, j \in I$ with $i \neq j$. Then the set $\left\{j, \bar{i}_{2 n+1}, \bar{i}_{2 n}, \ldots, \bar{i}_{3}, \bar{i}_{2}\right\}$ is a connection from $j$ to $i$.

Proof. Let us prove it by induction on $n$.

For $n=1$ we have that $i_{1}=i$ and $j \in \phi\left(\{i\}, i_{2}, i_{3}\right)$. Hence $j \in \mu\left(i, i_{2}, i_{3}\right)$ and by Lemma $2.1, i \in \mu\left(j, \bar{i}_{2}, \bar{i}_{3}\right) \subset \phi\left(\{j\}, \bar{i}_{2}, \bar{i}_{3}\right)=\phi\left(\{j\}, \bar{i}_{3}, \bar{i}_{2}\right)$. From here $\left\{j, \bar{i}_{3}, \bar{i}_{2}\right\}$ is a connection from $j$ to $i$.

Let us suppose that the assertion holds for any connection with $2 k+1$ elements and let us show it also holds for any connection $\left\{i_{1}, i_{2}, \ldots, i_{2 k+1}, i_{2 k+2}, i_{2 k+3}\right\}$ with $2 k+3$ elements.

If we denote $U:=\phi\left(\phi\left(\ldots \phi\left(\left\{i_{1}\right\}, i_{2}, i_{3}\right) \ldots\right), i_{2 k}, i_{2 k+1}\right)$ then we have that

$$
j \in \phi\left(U, i_{2 k+2}, i_{2 k+3}\right) \text {. }
$$

From here, Lemma 2.2 allows us to assert $\phi\left(\{j\}, \bar{i}_{2 k+2}, \bar{i}_{2 k+3}\right) \cap U \neq \emptyset$ and so we can take $h \in U$ such that

$$
h \in \phi\left(\{j\}, \bar{i}_{2 k+2}, \bar{i}_{2 k+3}\right) .
$$


Since $h \in U$ we have that $\left\{i_{1}, i_{2}, \ldots, i_{2 k}, i_{2 k+1}\right\}$ is a connection from $i$ to $h$ and so, by induction hypothesis, the set $\left\{h, \bar{i}_{2 k+1}, \bar{i}_{2 k}, \ldots, \bar{i}_{3}, \bar{i}_{2}\right\}$ is a connection from $h$ to $i$. This fact together with Equation (1) give us that

$$
i \in \phi\left(\phi\left(\ldots \phi\left(\phi\left(\{j\}, \bar{i}_{2 k+3}, \bar{i}_{2 k+2}\right), \bar{i}_{2 k+1}, \bar{i}_{2 k}\right) \ldots\right), \bar{i}_{3}, \bar{i}_{2}\right) .
$$

Hence $\left\{j, \bar{i}_{2 k+3}, \bar{i}_{2 k+2}, \ldots, \bar{i}_{3}, \bar{i}_{2}\right\}$ is a connection from $j$ to $i$ and the proof is complete.

Proposition 2.1. The relation $\sim$ in $I$, defined by $i \sim j$ if and only if $i$ is connected to $j$, is an equivalence relation.

Proof. The reflexive and symmetric character of $\sim$ are given by Definition 2.1 an Lemma 2.3 respectively. To check the transitivity character, observe that if we consider a couple of connections $\left\{i_{1}, \ldots, i_{2 m+1}\right\}$ and $\left\{j_{1}, \ldots, j_{2 n+1}\right\}$ from $i$ to $j$ and from $j$ to $k$ respectively, then the set $\left\{i_{1}, \ldots, i_{2 m+1}, j_{2}, \ldots, j_{2 n+1}\right\}$ is a connection from $i$ to $k$.

By the above Proposition we can introduce the quotient set

$$
I / \sim:=\{[i]: i \in I\},
$$

becoming $[i]$ the set of elements in $I$ which are connected to $i$.

For any $[i] \in I / \sim$ we define the linear subspace

$$
T_{[i]}:=\bigoplus_{j \in[i]} \mathbb{F} e_{j}
$$

Lemma 2.4. If $\left\langle T_{[i]}, T, T_{[j]}\right\rangle+\left\langle T, T_{[i]}, T_{[j]}\right\rangle+\left\langle T_{[j]}, T, T_{[i]}\right\rangle \neq 0$ for some $[i],[j] \in I / \sim$, then $[i]=[j]$ and $\left\langle T_{[i]}, T, T_{[j]}\right\rangle+\left\langle T, T_{[i]}, T_{[j]}\right\rangle+\left\langle T_{[j]}, T, T_{[i]}\right\rangle \subset T_{[i]}$.

Proof. Since $\left\langle T_{[i]}, T, T_{[j]}\right\rangle+\left\langle T, T_{[i]}, T_{[j]}\right\rangle+\left\langle T_{[j]}, T, T_{[i]}\right\rangle \neq 0$, there exists $u \in[i], w \in[j]$ and $v \in I$ such that

$$
0 \neq\left\langle e_{u}, e_{v}, e_{w}\right\rangle \in \mathbb{F} e_{l} \text { or } 0 \neq\left\langle e_{v}, e_{u}, e_{w}\right\rangle \in \mathbb{F} e_{l} \text { or } 0 \neq\left\langle e_{w}, e_{v}, e_{u}\right\rangle \in \mathbb{F} e_{l}
$$

for some $l \in I$.

In any case, we have that $l \in \mu(u, v, w)$. Hence $l \in \phi(\{u\}, v, w)$ and $\{u, v, w\}$ is a connection from $u$ to $l$. From here, the transitivity of $\sim$ gives us $i \sim l$ and we can deduce

$$
\left\langle T_{[i]}, T, T_{[j]}\right\rangle+\left\langle T, T_{[i]}, T_{[j]}\right\rangle+\left\langle T_{[j]}, T, T_{[i]}\right\rangle \subset T_{[i]} .
$$

We also have that $l \in \mu(w, v, u)$, so $l \in \phi(\{w\}, v, u)$ and $\{w, v, u\}$ is a connection from $w$ to $l$. Then we have that $j \sim l$ and conclude $[i]=[j]$.

Definition 2.2. Let $T$ be a triple system with multiplicative basis $\mathcal{B}$. It is said that a subtriple $S$ of $T$ admits a multiplicative basis $\mathcal{B}_{S}$ inherited from $\mathcal{B}$ if $\mathcal{B}_{S}$ is a multiplicative basis of $S$ satisfying $\mathcal{B}_{S} \subset \mathcal{B}$.

Proposition 2.2. For any $[i] \in I / \sim$, the linear subspace $T_{[i]}$ is an ideal of $T$ admitting a multiplicative basis inherited from the one of $T$.

Proof. Since $T=\bigoplus_{i \in I} \mathbb{F} e_{i}$ we clearly have $T=\bigoplus_{[j] \in I / \sim} T_{[j]}$ and so we can write

$$
\begin{gathered}
\left\langle T_{[i]}, T, T\right\rangle+\left\langle T, T_{[i]}, T\right\rangle+\left\langle T, T, T_{[i]}\right\rangle= \\
\left\langle T_{[i]}, T, \bigoplus_{[j] \in I / \sim} T_{[j]}\right\rangle+\left\langle T, T_{[i]}, \bigoplus_{[j] \in I / \sim} T_{[j]}\right\rangle+\left\langle\bigoplus_{[j] \in I / \sim} T_{[j]}, T, T_{[i]}\right\rangle \subset T_{[i]},
\end{gathered}
$$


being last inclusion consequence of Lemma 2.4. That is, any $T_{[i]}$ is actually an ideal of $T$. Finally, observe that the set $\left\{e_{j}: j \in[i]\right\}$ is a multiplicative basis of $T_{[i]}$ inherited from the basis $\mathcal{B}=\left\{e_{k}: k \in I\right\}$ of $T$.

Theorem 2.1. Let $T$ be a triple system admitting a multiplicative basis $\mathfrak{B}$. Then $T$ is the orthogonal direct sum of the the ideals

$$
T=\bigoplus_{[i] \in I / \sim} T_{[i]},
$$

admitting each $T_{[i]}$ a multiplicative basis inherited from $\mathfrak{B}$.

Proof. As in Proposition 2.2, the fact $T=\bigoplus_{i \in I} \mathbb{F} e_{i}$ gives us $T=\bigoplus_{[i] \in I / \sim} T_{[i]}$. Now, Proposition 2.2 and Lemma 2.4 complete the proof.

\section{THE MINIMAL COMPONENTS}

In this section our target is to characterize the minimality of the ideals which give rise to the decomposition of $T$ in Theorem 2.1 , in terms of connectivity properties in the set of indexes $I$.

Definition 3.1. An arbitrary triple system $T$ admitting a multiplicative basis $\mathcal{B}$ is called minimal if its only nonzero ideal admitting a multiplicative basis inherited from $\mathcal{B}$ is $T$.

Let us introduce the notion of $\mu$-multiplicativity in the framework of arbitrary triple systems admitting a multiplicative basis, in a similar way to the ones of closed-multiplicativity for Lie triple systems (see [10, 12, 15]), for split 3-Lie algebras (see [16]), or for different classes of algebras like graded associative algebras, split Leibniz algebras, split Poisson algebras, or split Lie color algebras (see $[13,14,17,18]$ for these notions and examples).

Definition 3.2. It is said that a triple system $T$ admits a $\mu$-multiplicative basis $\mathcal{B}=\left\{e_{i}\right\}_{i \in I}$ if it is multiplicative and given $i, j \in I$ such that $j \in \mu(i, x, y)$ for some $x, y \in I \dot{\cup} \bar{I}$ then $e_{j} \in\left\langle e_{i}, T, T\right\rangle+\left\langle T, e_{i}, T\right\rangle+\left\langle T, T, e_{i}\right\rangle$.

Examples of triple systems admitting $\mu$-multiplicative bases are those triple systems associated to (associative) matrix algebras, to semisimple finite-dimensional Lie algebras (over algebraically closed fields of characteristic 0 ), to semisimple separable $L^{*}$-algebras, to semisimple locally finite split Lie algebras or to the split Lie algebras considered in $[9, \S 3]$. We also can take as examples of triple systems with $\mu$-multiplicative bases to the associative triple systems $\mathcal{M}_{n \times m}(\mathbb{F}), n \neq m$, (see $\S 1$ ), to the meson triple systems described in $\S 1$, to the family of Jordan and Lie triple systems $\mathcal{N}$, (also described in $\S 1$ ), to the Lie triple systems considered in $[10,12,15]$, to the 3 -Lie algebras presented in [16] or to the Leibniz triple systems described in [2, Example 2.1].

Theorem 3.1. Let $T$ be an arbitrary triple system admitting a $\mu$-multiplicative basis $\mathfrak{B}=$ $\left\{e_{i}\right\}_{i \in I}$. Then $T$ is minimal if and only if the indexes set I has all of its elements connected.

Proof. Suppose $T$ is minimal. By Theorem 2.1, $T=T_{[i]}$ for some $[i] \in I / \sim$. Hence $[i]=I$ and so any couple of elements in $I$ are connected.

Conversely, consider a nonzero ideal $\mathfrak{I}$ of $T$ admitting a basis inherited from $\mathfrak{B}$. Then, for a certain $\emptyset \neq I_{\mathfrak{I}} \subset I$, we can write $\mathfrak{I}=\bigoplus_{j \in I_{\mathfrak{J}}} \mathbb{F} e_{j}$. Fix some $i_{0} \in I_{\mathfrak{I}}$ being then

$$
0 \neq e_{i_{0}} \in \mathfrak{I} \text {. }
$$


Let us show by induction on $n$ that if $\left\{i_{1}, \ldots, i_{2 n+1}\right\}$ is a connection from $i_{0}$ to some $j \in I$, then for any $k \in \phi\left(\phi\left(\cdots \phi\left(\left\{i_{1}\right\}, i_{2}, i_{3}\right) \ldots\right), i_{2 n}, i_{2 n+1}\right)$ we have that $0 \neq e_{k} \in \mathfrak{I}$.

Indeed, in case $n=1$, we get $k \in \phi\left(\left\{i_{1}\right\}, i_{2}, i_{3}\right)$ with $i_{1}=i_{0}$. Hence $k \in \mu\left(i_{0}, i_{2}, i_{3}\right)$, so, taking into account that $\mathfrak{I}$ is an ideal of $T$, by $\mu$-multiplicativity of $\mathfrak{B}$ and the Equation (2) we obtain $e_{k} \in \mathfrak{I}$.

Suppose now the assertion holds for each connection $\left\{j_{1}, \ldots, j_{2 h+1}\right\}$ from $i_{0}$ to any $r \in I$, and consider any connection $\left\{i_{1}, \ldots, i_{2 h+1}, i_{2 h+2}, i_{2 h+3}\right\}$ from $i_{0}$ to some $j \in I$. We know that for each $x \in U$, where $U:=\phi\left(\phi\left(\cdots \phi\left(\left\{i_{1}\right\}, i_{2}, i_{3}\right) \cdots\right), i_{2 h}, i_{2 h+1}\right)$, the element

$$
0 \neq e_{x} \in \mathfrak{I}
$$

Taking into account that the fact $k \in \phi\left(\phi\left(\cdots \phi\left(\left\{i_{1}\right\}, i_{2}, i_{3}\right) \ldots\right), i_{2 h+2}, i_{2 h+3}\right)$ implies

$$
k \in \phi\left(U, i_{2 h+2}, i_{2 h+3}\right),
$$

we have that $k \in \mu\left(x, i_{2 h+2}, i_{2 h+3}\right)$ for some $x \in U$. From here, the $\mu$-multiplicativity of $\mathfrak{B}$ and Equation (3) allow us to get $e_{k} \in \mathfrak{I}$ as desired.

Now, since given any $j \in I$ we know that $i_{0}$ is connected to $j$, we can assert by the above observation that $\mathbb{F} e_{j} \subset \mathfrak{I}$. We have shown

$$
T=\bigoplus_{j \in I} \mathbb{F} e_{j} \subset \mathfrak{I}
$$

and so $\mathfrak{I}=T$.

Theorem 3.2. Let $T$ be a triple system admitting a $\mu$-multiplicative basis $\mathfrak{B}$. Then $T$ is the orthogonal direct sum of the family of its minimal ideals

$$
T=\bigoplus_{k} \mathfrak{I}_{k}
$$

each one admitting a $\mu$-multiplicative basis inherited from $\mathfrak{B}$.

Proof. By Theorem 2.1 we have that $T=\bigoplus_{[i] \in I / \sim} T_{[i]}$ is the orthogonal direct sum of the ideals $T_{[i]}$, admitting each $T_{[i]}$ a multiplicative basis $\mathfrak{B}_{[i]}=\left\{e_{j}: j \in[i]\right\}$ inherited from $\mathfrak{B}$.

We wish to apply Theorem 3.1 to any $T_{[i]}$ so we are going to verify that the basis $\mathfrak{B}_{[i]}$ is a $\mu$-multiplicative basis and that $[i]$ has all of its elements $[i]$-connected (connected through connections contained in $[i] \dot{U} \overline{[i]})$.

We clearly have that $T_{[i]}$ admits to $\mathfrak{B}_{[i]}$ as a $\mu$-multiplicative basis as consequence of having a basis inherited from $\mathfrak{B}$ and the fact $\left\langle T_{[i]}, T, T_{[j]}\right\rangle+\left\langle T, T_{[i]}, T_{[j]}\right\rangle+\left\langle T, T_{[j]}, T_{[i]}\right\rangle=$ 0 when $[i] \neq[j]$.

Finally, consider any connection from $i$ to $j$

$$
\left\{i_{1}, i_{2}, \ldots, i_{2 n+1}\right\} \text {. }
$$

Let us observe that any $k \in \phi\left(\{i\}, i_{2}, i_{3}\right)$ satisfies $k \in[i]$ through the connection $\left\{i, i_{2}, i_{3}\right\}$. Then we have two possibilities:

- If $i_{2}, i_{3} \in I$, we also have, see Remark 2.1, that $k \in \phi\left(\left\{i_{2}\right\}, i, i_{3}\right)$ and $k \in$ $\phi\left(\left\{i_{3}\right\}, i, i_{2}\right)$. From here, the sets $\left\{i_{2}, i, i_{3}\right\}$ and $\left\{i_{3}, i, i_{2}\right\}$ are connections from $i_{2}$ to $k$ and from $i_{3}$ to $k$ respectively. Hence $i_{2}, i_{3} \in[i]$.

- If $i_{2}, i_{3} \in \bar{I}$, by Lemma 2.1 we have $i \in \phi\left(\{k\}, \bar{i}_{2}, \bar{i}_{3}\right)$. By arguing as above we get $\bar{i}_{2}, \bar{i}_{3} \in[i]$ and so $i_{2}, i_{3} \in \overline{[i]}$. 
By iterating this process we obtain that all of the elements in the connection (4) are contained in $[i] \dot{\cup} \overline{[i]}$. That is, $[i]$ has all of its elements $[i]$-connected. From the above, we can apply Theorem 3.1 to any $T_{[i]}$ so as to conclude $T_{[i]}$ is minimal.

It is clear that the decomposition $T=\bigoplus_{[i] \in I / \sim} T_{[i]}$ satisfies the assertions of the theorem.

\section{REFERENCES}

[1] Abdesselam, B.: The twisted Heisenberg algebra $e_{h, w}(H(4))$. J. Math. Phys. 38, no. 12, (1997), 60456060.

[2] Albeverio, S., Ayupov, S.A., Omirov, B.A. and Turdibaev, R.M.: Cartan subalgebras of Leibniz $n$-algebras. Comm. Algebra. 37 (2009), 2080-2096.

[3] Balogh, Z.: Further results on a filtered multiplicative basis of group algebras. Math. Commun. 12(2), (2007), 229-238.

[4] Bautista, R., Gabriel, P., Roiter, A.V. and Salmeron, L.: Representation-finite algebras and multiplicative basis. Invent. math. 81 (1985), 217-285.

[5] Bovdi, V.: On a filtered multiplicative basis of group algebras. Arch. Math. (Basel) 74(2) (2000), 81-88.

[6] Bovdi, V.: On a filtered multiplicative bases of group algebras. II. Algebr. Represent. Theory 6 (2003), no. 3, 353-368.

[7] Bovdi, V., Grishkov, A. and Siciliano, S.: Filtered multiplicative bases of restricted enveloping algebras. Algebr. Represent. Theory 14 (2011), no. 4, 601-608.

[8] Bovdi, V., Grishkov, A. and Siciliano, S.: On filtered multiplicative bases of some associative algebras. Algebr. Represent. Theory. DOI 10.1007/s10468-014-9494-7.

[9] Calderón, A.J.: On split Lie algebras with a symmetric root system. Proc. Indian Acad. Sci. (Math. Sci.) 118 (2008), no. 3, 351-356

[10] Calderón A.J.: On simple split Lie triple systems. Algbr. Represent. Theor. 12, (2009), 401-415.

[11] Calderón A.J.: Integrable roots in split Lie triple systems. Acta Mathematica Sinica 25, (2009), 1759-74.

[12] Calderón A.J.: On split Lie triple systems. Proc. Indian. Acad. Sci. (Math. Sci.) 119(2), (2009), 165-177.

[13] Calderón, A.J.: On graded associative algebras. Rep. Math. Phys. 69 (2012), no. 1, 75-86.

[14] Calderón, A.J.: On the structure of split non-commutative Poisson algebras. Linear and multilinear algebra. 60(7), (2012), 775-785.

[15] Calderón, A.J. and Forero, M.: On split Lie triple systems II. Proc. Indian Acad. Sci. (Math. Sci.) 120(2), (2010), 185-198.

[16] Calderón, A.J. and Forero, M.: Split 3-Lie algebras. J. Math. Phys. 52, (2011), 123503, 16 pp.

[17] Calderón, A.J. and Sánchez, J.M.: Split Leibniz algebras. Linear Algebra Appl. 436(6), (2012), 1648-1660.

[18] Calderón, A.J. and Sánchez, J.M.: On the structure of split Lie color algebras. Linear Algebra Appl. 436(2), (2012), 307-315.

[19] de la Mora, C. and Wojciechowski, P.J.: Multiplicative bases in matrix algebras. Linear Algebra Appl. 419 (2006), no. 2-3, 287-298.

[20] Jacobson, N.: Lie and Jordan triple system. Amer. J. Math. 71, no. 1, (1949), 149-170.

[21] Kupisch, H. and Waschbusch, J.: On multiplicative basis in quasi-Frobenius algebras. Math. Z. 186, (1984), 401-405.

[22] Ren, B., Ji Meng, D.: Some two steps nilpotent Lie algebras I. Linear Algebra Appl. 338, (2001), 77-98.

[23] Roiter A.V., Sergeichuk, V.V.: Existence of a multiplicative basis for finitely spaced module over an aggregate. Ukr. Math. J. 46(5), (1995), 604-617.

[24] Schue, J.R.: Hilbert space methods in the theory of Lie algebras. Trans. Amer. Math. Soc. 95 (1960), 69-80.

[25] Stumme, N.: The structure of locally finite split Lie algebras. J. Algebra. 220 (1999), 664-693.

E-mail address: a jesus.calderon@uca.es

E-mail address: javi.navarroiz@uca.es

E-mail address: txema. sanchez@uca.es

Department of Mathematics, FaCUlty of SCiEnCEs, University of CÁdiz, CAMpus de Puerto REAL, 11510, Puerto ReAl, CÁdiz, SPAin. 